\title{
Les Harpacticides du Val Piora
}

et note sur une anomalie de la furca chez Cyclops affinis Sars. par

\section{A. GR A E T E R.}

Avec la planche 10.

Fn comparant les familles des Copépodes d'eau douce, on remarque de suite un curieux antagonisme entre les Cyclopides et les Harpacticides en ce qui concerne leur nombre et leur distribution.

D'une part nous avons vu nos 150 espèces de Cyclops se réduire coup sur coup, jusqu'à arriver à un nombre de formes bien établies dépassant à peine la vingtaine! ${ }^{1}$

D'autre part nous voyons grandir de jour en jour le nombre dés Harpacticides. Il ne parait pas, sur cette famille, de publication qui n'augmente la liste d'un nouveau nom. De deux espèces de (anthocamptus (staphylimus et minutus) on en est arrivé pour les Harpacticides à plusieurs nouveaux genres et à un nombre d'espèces atteignant celui que nous venons de constater pour les Cyclops. ${ }^{2}$

${ }_{1}^{1}$ Sснмег. Deutschlands freilebende Süsswasser-Copepoden. I. Teil : Cyclopido. Bibliotheca zool. Heft 11, 1892, p. 189.

Riснавр. Recherches sur le système glandulaire et sur le système nerveux des Copépodes libres d'eau douce, suivies d'une revision des espèces de ce groupe, qui vivent en France. Ann. scienc. nat. zool. T. XII. 1891.

2 Мrazeck : Beitrag zur Kenntnis der Harpacticidenfauna des Süsswassers. Zool. Jahrb. Abteil. für Systematik Bd. VII. 1892. 
Ce dernier genre, au contraire, ne s'emrichit guère, malgré les nombreuses recherches faites dans toutes les contrées européennes. Dans les Iles Britanniques aussi bien qu’en Hongrie ou en Macédoine on ne retrouve que les formes déjà signalées dans l'Europe centrale. A une ou deux exceptions près, nous retrouvons ces mêmes formes en Afrique, en Australie, aux Etats-Unis, aux Philippines etc. Chaque nouvelle étude sur les faunes étrangères ne contribue qu'à accentuer toujours plus le cosmopolitisme de ce seul genre des Cyclopides d'eau douce.

En considérant ces faits, nous nous attendions a priori a rencontrer des conditions analogues pour la distribution verticale. En effet, jusqu'ici, les recherches méthodiques, opérées dans les hautes Alpes par ZscHoKKE, n’ont fait découvrir aucune nouvelle espèce de Cyclops. Par contre, sur les six Canthocamptus qu il a trouvés, trois ont été reconnus comme formes nouvelles par SchmerL qui les a décrites sous les noms de Canth. rhaticus, C. Zscholikei et C. cuspidatus. ${ }^{1}$

L'étude des Harpacticides du Val Piora que son explorateur a bien voulu nous confier, n’était donc pas dépourvue d'intérêt et les résultats obtenus, sans être abondants, ont montré que nous ne nous étions pas trompés dans nos prévisions. Nous avons constaté deux Canthocamptus pour le Val Piora, dont l'un se trouve être le Canthocamptus cuspidatus du Rhseticon et l'autre une espèce nouvelle. Jusqu'ici le C. cuspidatus n’avait été découvert que dans une source près de Partnun et le fait qu'il existe un second habitat sur le versant sud des Alpes, nous prouve clairement que nous n'avons pas affaire à des variétés locales, mais certainement à des formes distinctes, répandues sur un grand territoire.

${ }^{1}$ Schuem. Copepoden des Rhätition-Gebirges. Abhandl. d. Naturf. Gesellschaft zt Halle. Bd. XIX. 1893.

Sснмегl. Einige neue Harpacticidenformen des Süsswassers. Zeitschrift für Naturwissenschaft. Halle. Band $67,1894$. 
Nous n'avons pas grand chose à ajouter à la description faite par Schment, dans l'ouvrage précité.

La longueur de nos exemplaires, les soies de la furca nort comprises, varie de $0,61 \mathrm{~mm}$. à $0,65 \mathrm{~mm}$. pour les femelles ovigères, et 0,45 et 0,5 pour des mâles en copulation.

La description que SchyerL donne du dernier segment abdominal ne concorde pas avec ce que nous avons pu constater sur nos exemplaires. Ce segment porte, selon sichneIL, les mêmes ornements dans les deux sexes. Or nos exemplaires mâles ne possèdent à leur opercule anal que dix dents, soit la moitié moins que la femelle et la moitié du nombre que Somven indique dans son dessin de l'opercule mâle. Ceci nous montre à quel point l'on doit être prudent dans le choix des caractères.

Le second Canthocamptus trouvé par FunRuase n'a pu être identifié avec aucune autre forme, nous l'avons nommé Canth. unisetiger, en raison d'une particularité de sa furca. Il appartient au même groupe que le Canth. cuspidatus, mais se rattache plus particulièrement au Canthocamptus rhaticus.

Il s'en distingue surtout par son opercule anal. Le nombre des dents est égal, il est vrai, mais les dents de notre forme sont courtes, larges et se touchent entre elles par la base, de sorte que le contact n'est interrompu nulle part. Celles du C. rheticus, au contraire, sont longues, effilées; les bases ne se touchent pas, mais laissent une sorte de diastème entre elles.

Les antemnes antérieures nous fournissent une seconde différence. Le bâtonnet sensoriel qui, pour les espèces des Alpes rhétiques, atteint ou dépasse la fin du huitième article, c'est-à-dire la fin de l'antenne, dépasse à peine le pénultième article de notre nouvelle forme.

La branche interne des pattes natatoires de la première paire est biarticulée.

Les pattes de la cinquième paire ont le même aspect que celle des Canthocamptus grisons. Le deuxième article porte six appen- 
dices comme le Canthocamptus cuspidatus, et non pas cinq comme le C. rhaticus.

La parenté avec cette dernière espèce apparait de nouveau dans les dimensions et les contours généraux de la furca. Les bords internes sont garnis de deux rangées transversales de cils, comme chez le Canthocamptus rheticus.

Pourtant il est impossible de confondre la furca des deux espèces, car c'est justement dans cet organe que nous trouvons le caractère principal du Canth. unisetiger, celui qui le distingue de toutes les autres formes et qui lui a valu son nom.

Des deux grandes soies médianes qui terminent chacune des deux branches de la furca, le Canth. unisetiger n'en possèdequ'une seule. L'externe se trouve réduite à une minuscule proéminence. On pourrait attribuer cette lacune à quelque mutilation, mais le fait qu' elle se retrouved'une manière identique aux deux branches, nous montre que nous avons affaire à un caractère spécitique.

La longueur, les soies de la furca non comprises, est de 0,42 mm.

Nous espérons que de nouvelles recherches permettront de compléter cette description, faite d'après un seul exemplaire (femelle ovigère). Des pêches ultérieures nous feraient évidemment aussi connaitre de nouvelles formes; du moins, les expériences faites dans les Alpes rhétiques nous permettent de le croire. En effet, les premières recherches qu'on y fit ne domnèrent pas un seul représentant de notre genre, tandis que les suivantes produisirent trois espèces et que ce dernier nombre se trouve déjà doublé à la suite d'une troisième série de pêches.

SUR UNE ANOMALIE DE LA FURCA CHEZ LE

Cyclops affinis Sars.

Peu de temps après avoir fait l'étude du Canthocamptus unisetiger, nous avons rencontré dans un étang de Huningue un Cyclops dont les exemplaires femelles présentaient une curieuse anomalie. 
Comme on n’a point encore décrit de cas semblable chez aucun Copépode, et comme il se pourrait qu'il existât un certain rapport entre lui et le caractère essentiel du Canthocamptus, dont on vient de parler, nous en domnerons un croquis.

Le Cyclops affimis est assez rare. Aux environs de Bâle, nous l'avons trouvé en quatre endroits: dans le grand étang du Bambois entre Delle et Belfort, en France; dans les étangs de Bonfol au Jura Bernois et dans le Looweiher entre Zofingue et Olten, en Suisse: au Petit Huningue, dans un étang situé au-delà de la frontière, en Allemagne. Les exemplaires des trois premières localités ne se distinguaient par aucune particularité, ils étaient normaux et présentaient cette constance dans les moindres détails qui caractérise le genre Crclops et en fait l'antipode des Cladocères. Les rapports de la longueur des soies et de la furca notamment étaient les mêmes que ceux indiqués par RichaRD ${ }^{1}: 12$ (furca) 12, 35, 85. 8. Quant aux exemplaires du Cyclops affinis de Huningue, on en pourrait dire autant sans une irrégularité qui se trouve aux deux branches de la furca et qui consiste dans l'absence complète de la soie correspondant au terme 35 de la proportion de Richano. Le croquis représenté sur la planche montre très bien cette anomalie et rend superflue toute description plus détaillée. On voit tout de suite que l'analogie avec la furca du Canthocamptus. unisetiger n'est pas complète, le petit cone qui remplace la soie en question chez cette espèce, faisant entièrement défaut rhez notre Cyclops.

Il nous reste a mentionner un fait curieux, c'est que les exemplaires mâles ne présentent pas cette variation. Des 19 représentants de l'espèce, recueillis à Huningue, deux seulement possédaient un abdomen normal, - c'étaient les seuls individus mâles.

${ }_{1}$ Recision des espèces de Copépodes libres d'eau douce qui vicent en France. Ann. seienc. nat. zool. T. XII. 1891. 


\section{EXPLICATION DE LA PLANCHE 10.}

Fig. 1. Canthocamptus unisetiger nov. spec. Patte de la cinquième paire. Gross. $\times 700$.

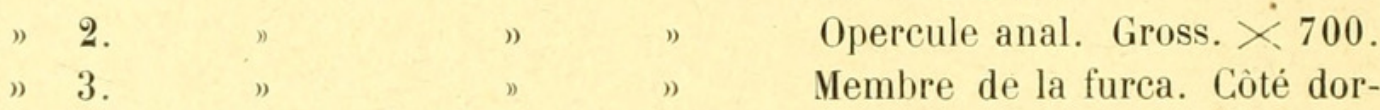
sal. Gross $\times 700$.

gr. s. ext. = grande soie externe.

gr. s. int. = grande soie interne.

C. ext. = coté externe.

s. d. = soie dorsale.

C. int. = côté interne.

Fig. 4. Cyclops affinis Sars. exempl. $q$ de Huningue.

t. = tube digestif.

s. a. = segment antépénultième de l'abdomen.

s. p. = segment pénultième de l'abdomen.

tels. = telson ou dernier segment de l'abdomen.

op. = opercule anal (moitié supra-anale du telson).

r. = rétracteur de l'anus.

c. = rangée de cils anales.

a. $=$ anus.

br. = branche de la furca ou extrémité du segment pénultième de l'abdomen.

s. $8=$ soie 8 du rapport de Richard.

s. $85=$ soie 85 du ” ” ”

v. = vide laissé par la soie $3 \check{\text {. }}$.

s. $12=\operatorname{soie} 12$. 


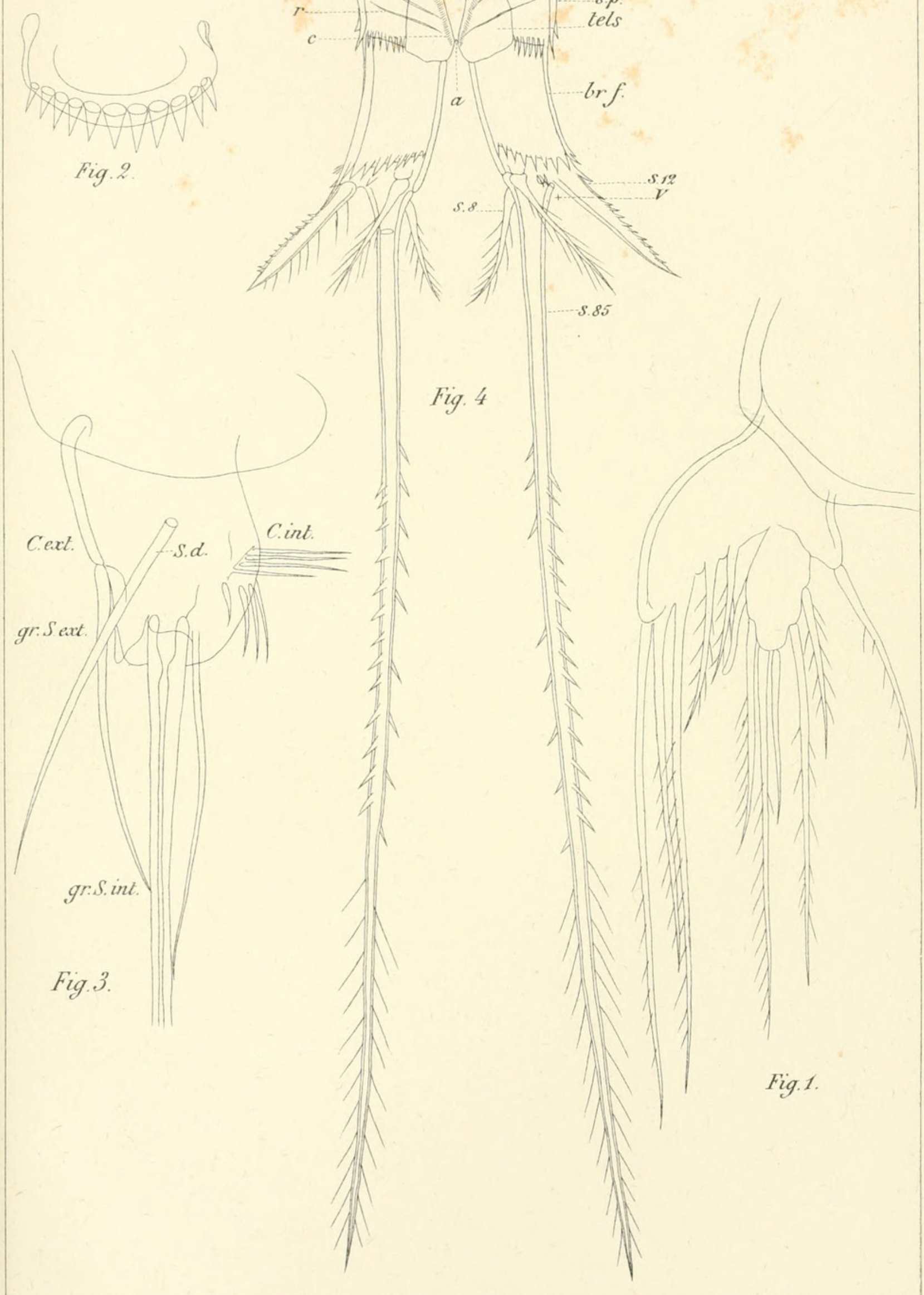




\section{$2 \mathrm{BHL}$ Biodiversity Heritage Library}

Graeter, A. 1899. "Les Harpacticides du val Piora et note sur une anomalie de la furca chez Cyclops affinis Sars." Revue suisse de zoologie 6(2), 363-367. https://doi.org/10.5962/bhl.part.75150.

View This Item Online: https://www.biodiversitylibrary.org/item/39595

DOI: https://doi.org/10.5962/bhl.part.75150

Permalink: https://www.biodiversitylibrary.org/partpdf/75150

\section{Holding Institution}

MBLWHOI Library

\section{Sponsored by}

MBLWHOI Library

\section{Copyright \& Reuse}

Copyright Status: NOT_IN_COPYRIGHT

This document was created from content at the Biodiversity Heritage Library, the world's largest open access digital library for biodiversity literature and archives. Visit BHL at https://www.biodiversitylibrary.org. 